\title{
Selective Metal-Site-Guided Arylation of Proteins
}

\author{
Jens Willwacher, Ritu Raj, Shabaz Mohammed, Benjamin G. Davis* \\ Department of Chemistry, University of Oxford, Chemistry Research Laboratory, Mansfield Road, Oxford OX1 3TA, UK \\ Supporting Information Placeholder
}

\begin{abstract}
We describe palladium-mediated $S$-arylation that exploits natural metal-binding motifs to ensure high siteselectivity for a proximal reactive residue. This allows the chemical identification not only of proteins that bind metals but the environment of the metal-binding site itself through proteomic analysis of arylation sites. The transformation is easy to perform under standard conditions, does not require the isolation of a reactive Ar-Pd complex, is broad in scope and is applicable in cell lysates as well as to covalent inhibition/modulation of metaldependent enzymatic activity.
\end{abstract}

Post-translational modification is nature's method to decorate proteins (typically enzymatically) with structurally diverse functional 'switches' and recognition sites. ${ }^{1}$ Its advantage over the currently available chemical 'toolbox' for protein chemistry is its ability to carry out such reactions in a highly site-selective manner. ${ }^{2}$ Whilst this is, in part, achieved via chemoselectivity, it is often also guided by mutual recognition of secondary structure in either the protein substrate or the modifying enzyme catalyst. This guides regioselection and enables selectivity for certain residues, often related to or guided by function. Whilst regioselectivity directed simply by accessibility has proved a potentially successful approach in chemical protein modification, ${ }^{3}$ the ability to direct a protein-modifying chemical catalytic centre by virtue of functionally inherent motifs might allow regioselection guided by endogenous features in a manner that partially mimics nature's approach. Here we show, as a proof-of-principle, a designed method for site-selective protein modification that appears to rely, at least in part, upon natural metal-binding.

Several strategies for chemical protein modification have been developed; ${ }^{4,5}$ a classic variant relies on (sometimes partial) chemoselectivity of certain natural side-chains (e.g. lysine (Lys) / cysteine (Cys), Scheme 1a). ${ }^{6}$ For example, the amine/thiol groups of these residues can exhibit higher nucleophilicity towards some electrophiles. Most of these methods rely on solvent accessibility/exposure of particular Lys- or Cys-residues for regioselectivity, and often generate heterogeneous product mixtures. To reduce such heterogeneity in classical protein-conjugates, conceptually different site-selective modification strategies have emerged. These often rely on the incorporation of functional groups with beneficial reactivity ('tags') for improved chemoselection and hence regioselection, if reaction is complete. These include genetically-, enzymatically- and chemically- installed unnatural amino acids (UAAs) $)^{7,8}$ with 'bioorthogonal' reactivity and enhanced chemoselectivity with an appropriate reagent (Scheme 1b). ${ }^{9,10}$ Metal-mediated examples include azidealkyne 'cycloadditions', ${ }^{11,12}$ cross-couplings, ${ }^{13}$ and olefin crossmetathesis. ${ }^{14}$ Elegant, prior, metal-guided covalent protein modifications have typically ${ }^{15}$ exploited unnatural metalbinding peptide sequences at the termini of proteins, such as oligo-aspartate, ${ }^{16,17}$-histidine ${ }^{18-20}$ or -cysteine ${ }^{21}$ motifs. However, covalent protein modifications based on naturally occurring motifs to achieve site-selectivity are rare. ${ }^{22}$ The need for programmed genetic incorporation of unnatural sequences at restricted locations prohibits their use as a tool for $a$ priori identification of metal-binding proteins. We therefore set out to develop a conceptually different approach for siteselective protein modification by relying on endogenous metal-binding motifs (Scheme 1c). If successful, this would not only enable labeling of metal-binding proteins in vitro but also identification in cell lysates (e.g. through direct attachment of 'pull-down handles').

Scheme 1. Established strategies compared to metalsite guided protein modification.

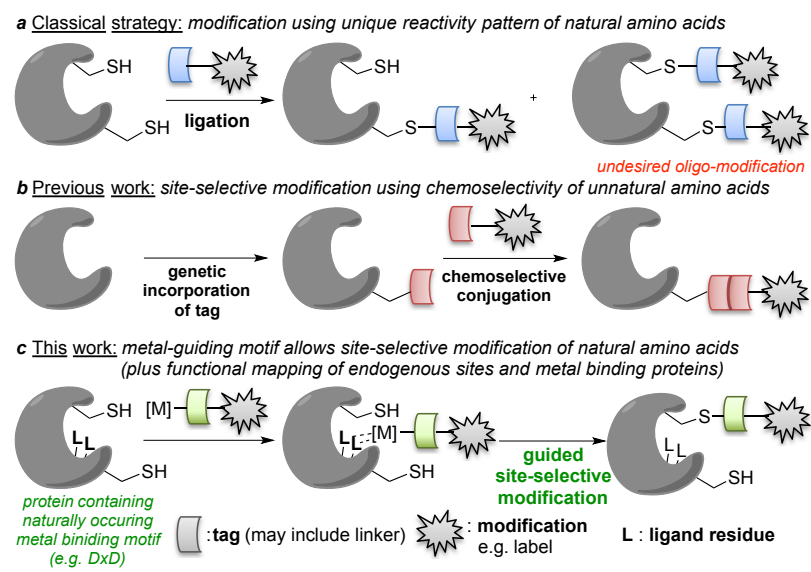

One form of common, naturally-occurring metal-binding motif is that found in enzyme active sites that enable metaldependent catalysis. We reasoned that these could direct a reactive metal complex into a given protein, thereby guiding selective reaction. Our proposed protein modification thus relied on two characteristic structural patterns: a) a metalbinding site that would steer the reactive complex; and b) a proximal, reactive amino-acid residue to react with the metal complex resulting in covalent modification. Class $\mathrm{A}^{23}$ glycosyltransferases (GTs) are archetypal metal-dependent enzymes and we chose mannosylglycerate synthase (MGS), originally isolated from the thermophilic bacteria Rhodothermus marinus, ${ }^{24}$ as a model system. MGS was the first mannosyltransferase to be fully structurally characterized $^{25}$ and displays a well-examined ${ }^{26}$ metal binding profile. GT-A folds contain a common DxD sequence as a metalbinding motif ${ }^{27}$; in MGS it is D10oA101D102 (PDB 2BO6). ${ }^{26}$ We speculated that this could steer an M(II)-Aryl species generated in situ into the active site, allowing it to react with 
Scheme 2. Development, analysis and scope of guided site-selective, Pd-mediated, cysteine-arylation.

a General reaction scheme (optimized conditions)
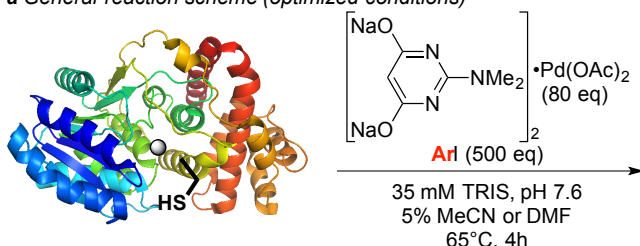

MGS $(1.0 \mathrm{mg} / \mathrm{mL})$

MW: 47514

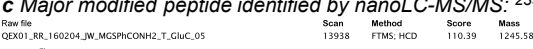

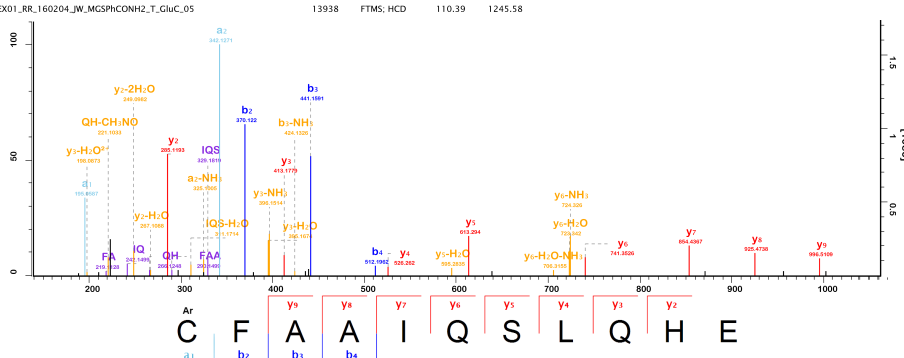

Representative LC-MS spectrum of crude reaction mixture $(A r=2)$ : ion series and deconvoluted spectra

d Scope of site-selective cysteine S-arylation with respect to aryl iodide. *: 250 eq. of Arl were used

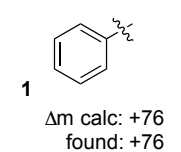

found: +76 conversion: $83 \%$

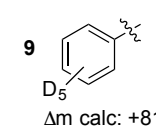

$\Delta$ m calc: +81 found: +79

conversion: $77 \%$

15 है

$\Delta \mathrm{m}$ calc: +77

found: +79

conversion*: $24 \%$

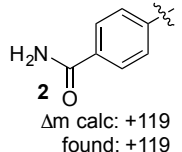

found: +119

conversion: $\mathbf{8 8 \%}$

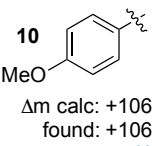

found: +106

conversion: $40 \%$

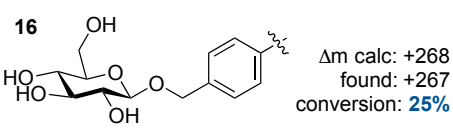

a range of potential residues, depending on reactivity \& mechanism, including proximal Cys233 thiolate (Sch. 1c). ${ }^{28}$

We first investigated several transition metal precatalysts ${ }^{13,29,30}$ in combination with iodobenzene for modifying MGS-His $_{6}$. Major emphasis was placed on palladium ${ }^{31}$ as a suitable metal mediator, as previous studies have shown its versatility in aqueous Suzuki- ${ }^{13,32}$ and Sonogashira-coupling reactions..$^{33}$ After a screen of a variety of systems, $\mathrm{Pd}(\mathrm{OAc})_{2}$ ligated with the disodium salt of $N, N$-dimethyl-2-amino-4,6dihydroxypyrimidine (DM-ADHP) proved most reactive at $65^{\circ} \mathrm{C}$ and was used as a precatalyst (Scheme 2a). Other precatalysts such as $\mathrm{Pd}\left(\mathrm{OC}(\mathrm{O}) \mathrm{CF}_{3}\right)_{2}, \mathrm{Pd}(\mathrm{OAc})_{2}$, or other watersoluble palladium pre-catalysts were unreactive and using these only unmodified MGS was obtained (see SI). At ambient temperature or $37^{\circ} \mathrm{C}$, only unmodified protein was detected by LC-MS analysis (see SI). This unique performance of DM-ADHP as ligand suggested that at least one molecule of ligand was still attached to the $\mathrm{Pd}(\mathrm{II})$-Aryl species during the reaction or was necessary to deliver the Pd-complex to the guiding site. Independently, Buchwald et al. have also elegantly shown that Pd-mediated cysteine $S$-arylation can be rendered general by tuning the surrounding ligand. ${ }^{34}$ Under our optimized conditions, only a single product was observed in $>85 \%$ conversion after $4 \mathrm{~h}$ (Scheme $2 \mathrm{~b}$ ). Consistent with our selectivity hypothesis, potential di- or oligomodified species were not detected by LC-MS despite other potential modifiable sites (e.g. Cys34, Cys209, Cys305); notably $\mathrm{C}_{305}$ is the most solvent exposed but does not arylate,
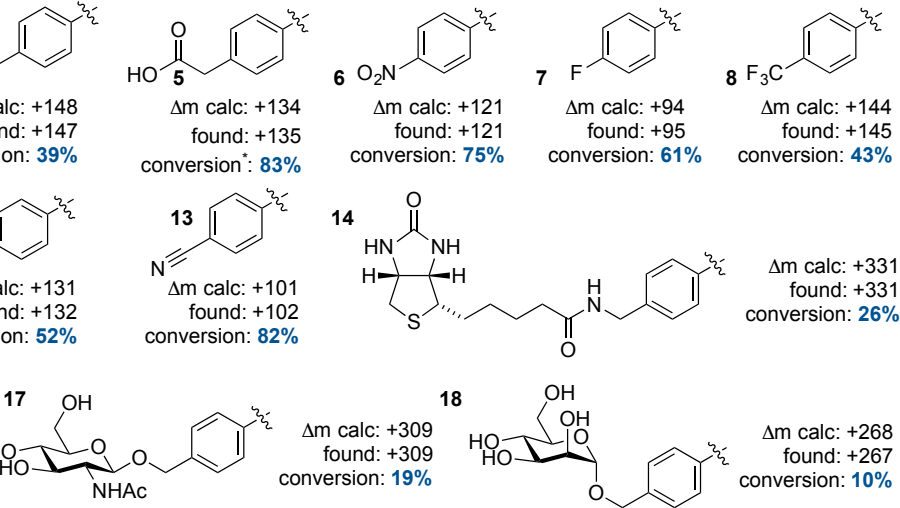

further suggesting regioselectivity directed by protein structure and aryl-Pd species. Longer reaction times led only to loss of protein through degradation / precipitation pathways.

The site of the modification was examined by LC-MS/MS analysis after in-gel digest with trypsin/endoproteinase GluC. This confirmed $\mathrm{C}_{233}$ as the major reaction site (Scheme 2c). Modified vs unmodified Cys sites were also established semiquantitatively through a carbamidomethylation strategy (see SI); this not only confirmed $\mathrm{C}_{23} 3$ as the primary reaction site but also highlighted the retained high reactivity of the Cys residues (which were unmodified by our metal-guided process) to non-directed Cys alkylation chemistry. To further test these sites of reactivity, we prepared mutants in which potentially reactive Cys were exchanged for Ala $\left(\mathrm{C}_{34} \mathrm{~A}\right.$, C209A, C233A). The reaction outcome remained unchanged with the C34A or C209A (Table 1, entry 2-3), whereas no product $(<5 \%)$ was detected for the $\mathrm{C}_{23} \mathrm{~A}$ a variant, confirming the high site-selectivity of the $S$-arylation (entry 4).

Mutagenesis was also used to probe the dependence of this site-selective cysteine arylation on the potentially important sites within the protein. Ala-scanning mutational analysis revealed significant effects (SI Figure S9) only from combined mutagenesis of D1oo, D102 that comprise the DxD and $\mathrm{T}_{139}$ (found in its accessory ion-engagement site ${ }^{25}$, entries $10,11,13)$. Although other backbone interactions that cannot be 'scanned' in this way likely also contribute, the partial role of this combined site was confirmed by triple mutation (entries 14,15 ) that reduced modification to $<\sim 30 \%$; other puta- 
tive binding/basic residues, e.g. H217, R131, had essentially no effect (Table 1). It should be noted that detailed analysis (see SI) of the structure MGS shows that D10o, D102, T139 contribute to part of the metal/ion-binding site; remaining backbone interactions and protein shell may explain retained residual directing activity of even triple DDT $\rightarrow$ AAA mutants.

Table 1. Effect of host mutations on $S$-arylation

\begin{tabular}{ccccc}
\hline \multirow{2}{*}{ entry } & protein & \multicolumn{3}{c}{ \% S-arylation ${ }^{a}$} \\
1 & His $_{6}$ MGS-WT & 75 & 58 & 49 \\
2 & C34A & 71 & 49 & 53 \\
3 & C209A & 75 & 55 & 46 \\
4 & C233A & $<5$ & $<5$ & $<5$ \\
5 & D100A & 72 & 57 & 51 \\
6 & D102A & 66 & 49 & 45 \\
7 & H217A & 75 & 60 & 51 \\
8 & D100A H217A & 58 & 35 & 58 \\
9 & D102A H217A & 59 & 43 & 51 \\
10 & D100A D102A & 42 & 29 & 36 \\
11 & D100A D102A H217A & 48 & 33 & 39 \\
12 & R131A & 66 & 43 & 55 \\
13 & T139A & 48 & 44 & 31 \\
14 & D100A D102A T139A & 30 & 34 & 27 \\
15 & D100A D102A T139A & 35 & 40 & 42 \\
16 & H217A & 65 & 38 & 52 \\
\hline${ }^{a}$ LC-MS conversion after 3h under conditions of Scheme 2.
\end{tabular}

As poly-His sites have been used to guide other protein chemistries, $^{18-20}$ we tested endogenous $v s$ such unnatural sites. The out-competing, directing effect of the identified metal/ion-binding D10o-D102-T139 motif over artificial (i.e. $\mathrm{His}_{6}$ ) motif was suggested by the essentially negligible influence of $\mathrm{His}_{6}$ in MGS (entry 16). The role of the DDT motif was further supported by lower arylation rates for the AAA mutant and by the attempted modification of several different proteins that bear accessible Cys but no nearby metalbinding sites; from these only unmodified proteins were recovered (see SI). Together, these data suggested an important role for the endogenous motif (Scheme 1c).

Next, we tested the scope of the reaction beyond $\mathrm{Ar}=\mathrm{Ph}$ towards functionalized aryls $(\mathbf{1}-\mathbf{1 8}$, Scheme $2 \mathrm{~d})$, including those containing useful labels and handles. The reaction is tolerant of a variety of different aryl iodides bearing reactive as well as biochemically-useful functional groups (Scheme 2d). Accordingly, proteins can be labelled with fluoro- $(\mathbf{7}, \mathbf{8})$ or deutero-labelled (9) groups and sugar (e.g. Glc- 16, GlcNAc- 17, Man-18), biotin- (14) or azide- (12) bearing moieties enabling potential subsequent detection, further modification or purification by affinity chromatography. Observed conversions varied in a manner that may reflect solubility in buffered aqueous media \& potentially differing abilities to enter the regions proximal to the metal-binding motif (although no clear trends supporting e.g. hydrophobic interaction were observed).

We also examined the capacity of this method to modulate protein (e.g. enzymatic) activity. The use of directed, covalent enzyme inhibition strategies has seen a resurgence in recent years ${ }^{36}$ and several enzyme target families ${ }^{37}$ contain conserved reactive residues, such as the Cys that we find here. Our identified site-selective $S$-arylation modified residue Cys233 in close proximity to the active site of MGS. Since metal binding sites also often coincide or abut active sites, we considered that this could enable active-site-directed, metal-mediated modulation/inhibition. Strikingly, evaluation of the transferase activity ${ }^{25,35}$ of representative $\mathrm{C}_{233}-\mathrm{S}$ arylated MGS variants revealed modification-dependent inhibition of MGS's endogenous enzymatic metabolic role (generation of stress protectant mannosylglycerate, Table 2). This vitally confirmed two key aspects: i) the arylation conditions are apparently benign with respect to global protein structure (here giving inhibited but measurable activity consistent with folded protein); and ii) proof-of-principle, structure-varied, covalent inhibition of a model GT. Indeed, variation of both size, functional group and the inclusion of biomimetic motifs (e.g. glycans that might additionally engage the active site) in the aryl moieties allowed the development of quite potent $(\sim 70-80 \%$ at $\sim 2 \mathrm{mM}, 200 \mu \mathrm{M}$ substrate) inhibition. A good correlation between level of inhibition and modification was observed in most cases, consistent with effective blocking of the active site (Table 2). Interestingly, Glc-bearing 16 showed relative levels of inhibition higher than others; Glc is a recognized sugar substrate for $\mathrm{MGS}^{25}$ and we tentatively assign this to potential site-to-site engagement of the Glc moiety by MGS.

Table 2. Covalent inhibition of MGS ${ }^{a}$

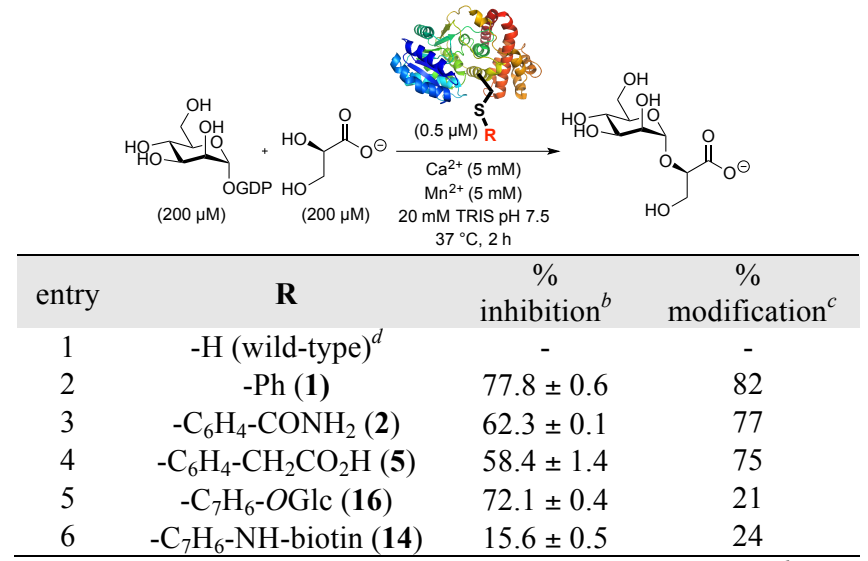

${ }^{a}$ average of two following arylation with $\sim 2 \mathrm{mM}$ reagent. ${ }^{b}$ from GDP release ${ }^{35}$ verified by $\mathrm{MS}^{25}$ (see SI). ${ }^{c}$ estimated by LC-MS. ${ }^{d}$ unmodified MGS subjected to identical conditions without ArI.

Finally, we were further able to show that this method is suitable for the a priori identification of other metaldependent proteins, even in complex mixtures (Figure 1). In this context, purified MGS as well as the heat-shocked crude cell lysates of MGS over-expression were treated with different $\mathrm{Pd}(\mathbf{L 2})_{2}(\mathrm{OAc})_{2}$ and biotin-aryl (14) iodide concentrations (along with associated controls) and visualized using antibiotin antibody. These enabled the detection of a metalbinding protein with a molecular weight of $\sim 60 \mathrm{kDa}$ (Figure 1, lanes G-I). In-gel tryptic digest allowed this band to be identified as the $60 \mathrm{kDa}$ chaperonin GroEL, ${ }^{38}$ a thermophilic, ATP-dependent protein that assists folding during the last steps of protein biosynthesis. In line with our designed method, GroEL binds $\mathrm{Mg}^{2+}$ through an aspartate-based motif. ${ }^{39,40}$ Mapping of modified GroEL by MS/MS (see SI) revealed Cysiz 8 as the primary site for arylation; notably, direct interaction of Cysiz 8 with the Mg•ATP binding site has been previously demonstrated..$^{41}$ The method was further verified under differing conditions, such as no overexpression or overexpression of a different protein, and gave essentially similar results (see SI). As well as being able to visualize identified proteins in this way, we were also able to demon- 
strate biotin-mediated affinity extraction using the attached biotin moiety found in $\mathbf{1 4}$ (see SI).

Figure 1. Identification of metal-binding proteins in crude cell lysate. SDS-PAGE (left) and Western blot stained with anti-Biotin antibody (right).

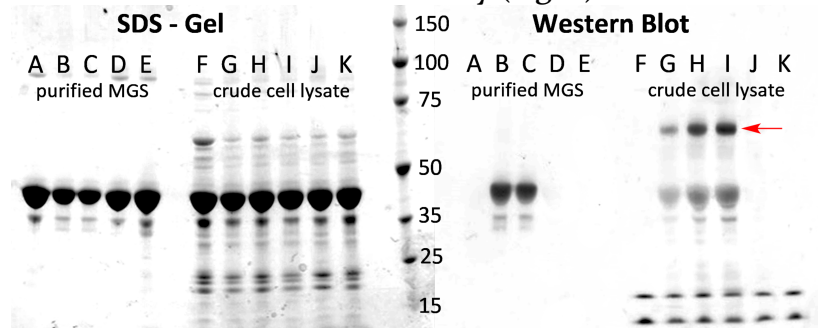

Reacn. 35 mM TRIS pH7.6, $67^{\circ} \mathrm{C}, 3$ h. Lanes: A MGS; B $2 \mathrm{mM}$ [Pd], 10 mM 14-I; C 2 mM [Pd], 5 mM 14-I; D no [Pd], 5mM 14I; E $2 \mathrm{mM}$ [Pd], $10 \mathrm{mM}$ PhI; F lysate; G 1mM [Pd], $5 \mathrm{mM} \mathrm{14-I;}$ H $2 \mathrm{mM}$ [Pd], $10 \mathrm{mM}$ 14-I; I 4 mM [Pd], $10 \mathrm{mM}$ 14-I; J no [Pd], 10 mM 14-I; K 2 mM Pd, 10 mM PhI. Red arrow: excised bands.

In conclusion, we have developed Pd-mediated siteselective $S$-arylation that exploits endogenous metal-binding motifs. In contrast to previous Pd-mediated bionconjugation reactions, ${ }^{34,42,43}$ the isolation of a preformed Pd-aryl complex was not necessary and renders this protocol easy to implement. It also enables regioselective differentiation to enhance product homogeneity in proteins bearing multiple Cys. We suggest that this work has established three proofsof-principle: firstly, not only that endogenous metal binding sites may be used to guide site-selective, metal-mediated modification (Scheme 1c) but secondly, since active sites of several enzymes (e.g. GTs) contain such binding motifs, it provides the opportunity for active-site-directed covalent modulation/inhibition of activity. Here, we show examples of covalent inhibition of GTs ${ }^{44}$ which inspires future investigations. Thirdly, this selectivity was demonstrated to be sufficient to allow protein-selective modification based on the presence of such an endogenous metal-binding motif (a selectivity that we tested here within complex cellular milieu). Thus, the method can also be used for a priori labeling, detection and identification of metal-dependent proteins by employing e.g. biotin-labeling in cell lysates. We have shown reaction here of quite different directing motifs in proteins suggesting generality based on metal or metal/ion binding; we cannot, of course, exclude other directing factors. We should note too that the conditions (e.g. temperature, reagents) may well be incompatible with certain proteins and future studies will focus on exploring breadth and altered reagent conditions to delineate such limitations further. The harsher reaction conditions for S-arylation as compared to e.g. Suzuki reactions ${ }^{13}$ on proteins are likely a consequence of the more demanding nature of the Pd-mediated C-S bond forming process; ${ }^{45}$ higher reaction temperature may also enable critical protein flexibility to allow interaction between targeted Cys residue and the metal binding site. We believe that the concept presented herein might represent a useful additional way to achieve site-selective protein modifications (and associated uses) without the need for incorporating either UAAs or unnatural motifs.

\section{Supporting Information}

Additional information on optimization, detailed experimental procedures, LC-MS and MS/MS spectra are included in the SI. The authors declare no competing financial interests.
Corresponding Author ben.davis@chem.ox.ac.uk ACKNOWLEDGMENT

We thank the German Academic Exchange Service (JW), \& the Felix Foundation (RR) for financial support and Drs C. Fehl, A. Fernandez-Tejada, Y. Geng, Mr S. Galan for helpful discussions.

(1) Walsh, C. T. Posttranslational Modification of Proteins: Expanding Nature's Inventory; Roberts and Company: Englewood, Colorado, 2006.

(2) Sletten, E. M.; Bertozzi, C. R. Angew. Chem. Intl Ed. 2009, 48, 6974.

(3) van Kasteren, S.I.; Kramer, H.; Jensen, H.; Campbell, S.; Kirkpatrick, J.; Oldham, N. J.; Anthony, D. C.; Davis, B. G. Nature 2007, 446, 1105.

(4) Boutureira, O.; Bernardes, G. J. L. Chem. Rev. 2015, 115, 2174.

(5) Spicer, C. D.; Davis, B. G. Nat. Commun. 2014, 5.

(6) Chalker, J; Bernardes, G; Lin, Y; Davis, B Chem Asian J 2009, 4, 630

(7) Young, T. S.; Schultz, P. G. J. Biol. Chem. 2010, 285, 11039.

(8) Wals, K.; Ovaa, H. Front. Chem. 2014, 2, 15.

(9) Kim, C.; Axup, J.; Schultz, P.G. Curr Opin Chem Biol 2013, 17, 412.

(10) Lang, K.; Chin, J. W. Chem. Rev. 2014, 114, 4764.

(11) Tornøe, C.; Christensen, C.; Meldal, M. J Org Chem 2002, 67, 3057.

(12) Rostovtsev, V. V.; Green, L. G.; Fokin, V. V.; Sharpless, K. B. Angew. Chem. Intl Ed. 2002, 41, 2596.

(13) Chalker, J.; Wood, C.; Davis, B.G. J Am Chem Soc 2009, 131, 16346

(14) Lin, Y. A.; Chalker, J. M.; Floyd, N.; Bernardes, G. J. L.; Davis, B. G. J. Am. Chem. Soc. 2008, 130, 9642 .

(15) Uchinomiya, S.; Ojida, A.; Hamachi, I. Inorg. Chem. 2014, 53, 1816. (16) Nonaka, H.; Fujishima, S.-H.; Uchinomiya, S.-H.; Ojida, A.; Hamachi, I. Bioorg. Med. Chem. Lett. 2009, 19, 6696.

(17) Ojida, A.; Honda, K.; Shinmi, D.; Kiyonaka, S.; Mori, Y.; Hamachi, I. J. Am. Chem. Soc. 2006, 128, 10452.

(18) Uchinomiya, S.; Nonaka, H.; Fujishima, S.; Tsukiji, S.; Ojida, A.; Hamachi, I. Chem. Commun. 2009, 5880.

(19) Hauser, C. T.; Tsien, R.Y. Proc Natl Acad Sci USA 2007, 104, 3693. (20) Rosen, C. B.; KodalAnne, L. B.; Nielsen, J. S.; Schaffert, D. H.; Scavenius, C.; Okholm, A. H.; Voigt, N. V.; Enghild, J. J.; Kjems, J.; Tørring, T.; Gothelf, K. V. Nat Chem 2014, 6, 804.

(21) Griffin, B. A.; Adams, S. R.; Tsien, R. Y. Science 1998, 281, 269.

(22) Hayashi, T.; Yasueda, Y.; Tamura, T.; Takaoka, Y.; Hamachi, I. J. Am. Chem. Soc. 2015, 137, 5372.

(23) Lairson, L. L.; Henrissat, B.; Davies, G. J.; Withers, S. G. Annu Rev Biochem 2008, 77, 521 .

(24) Martins, L. g. O.; Empadinhas, N.; Marugg, J. D.; Miguel, C.; Ferreira, C.; Costa, M. S. d.; Santos, H. J. Biol. Chem. 1999, 274, 35407.

(25) Flint, J.; Taylor, E.; Yang, M.; Bolam, D. N.; Tailford, L. E.; Martinez-Fleites, C.; Dodson, E. J.; Davis, B. G.; Gilbert, H. J.; Davies, G. J. Nat. Struct. Mol. Biol. 2005, 12, 608 .

(26) Nielsen, M.; Suits, M. D. L.; Yang, M.; Barry, C. S.; MartinezFleites, C.; Tailford, L. E.; Flint, J. E.; Dumon, C.; Davis, B. G.; Gilbert, H. J.; Davies, G. J. J. Biol. Chem. 2011, 286, 15155.

(27) Breton, C.; Šnajdrová, L.; Jeanneau, C.; Koča, J.; Imberty, A. Glycobiology 2006, 16, 29R.

(28) Lee, C. F.; Liu, Y. C.; Badsara, S. S. Chem. Asian J. 2014, 9, 706.

(29) Dumas, A.; Spicer, C. D.; Gao, Z.; Takehana, T.; Lin, Y. A.; Yasukohchi, T.; Davis, B. G. Angew. Chem. Intl Ed. 2013, 52, 3916.

(30) Gao, Z; Gouverneur, V; Davis, B. J Am Chem Soc 2013, 135, 13612.

(31) Yang, M.; Li, J.; Chen, P. R. Chem. Soc. Rev. 2014, 43, 6511.

(32) Spicer, C. D.; Davis, B. G. Chem. Commun. 2011, 47, 1698.

(33) Li, N; Lim, R; Edwardraja, S; Lin, Q. J Am Chem Soc 2011, 133, 15316

(34) Vinogradova, E. V.; Zhang, C.; Spokoyny, A. M.; Pentelute, B. L.; Buchwald, S. L. Nature 2015, 526, 687.

(35) Wu, Z. L.; Ethen, C. M.; Prather, B.; Machacek, M.; Jiang, W. Glycobiology 2011, 21, 727.

(36) Singh, J.; Petter, R. C.; Baillie, T. A.; Whitty, A. Nat Rev Drug Discov 2011, 10, 307.

(37) Zhang, J.; Yang, P. L.; Gray, N. S. Nat Rev Cancer 2009, 9, 28.

(38) Braig, K.; Otwinowski, Z.; Hegde, R.; Boisvert, D. C.; Joachimiak, A.; Horwich, A. L.; Sigler, P. B. Nature 1994, 371, 578.

(39) Wang, J.; Boisvert, D. C. J. Mol. Biol 2003, 327, 843.

(40) Boisvert, D. C.; Wang, J.; Otwinowski, Z.; Norwich, A. L.; Sigler, P. B. Nat Struct Mol Biol 1996, 3, 170.

(41) Bochkareva, E; Horovitz, A; Girshovich, A. J Biol Chem 1994, 269, 44

(42) Simmons, R.; Yu, R.; Myers, A. G. J Am Chem Soc 2011, 133, 15870

(43) Cheng, G.; Lim, R.; Li, N.; Lin, Q. Chem. Commun. 2013, 49, 6809.

(44) Tedaldi, L.; Wagner, G. K. MedChemComm 2014, 5, 1106.

(45) Kondo, T.; Mitsudo, T. Chem. Rev. 2000, 100, 3205. 


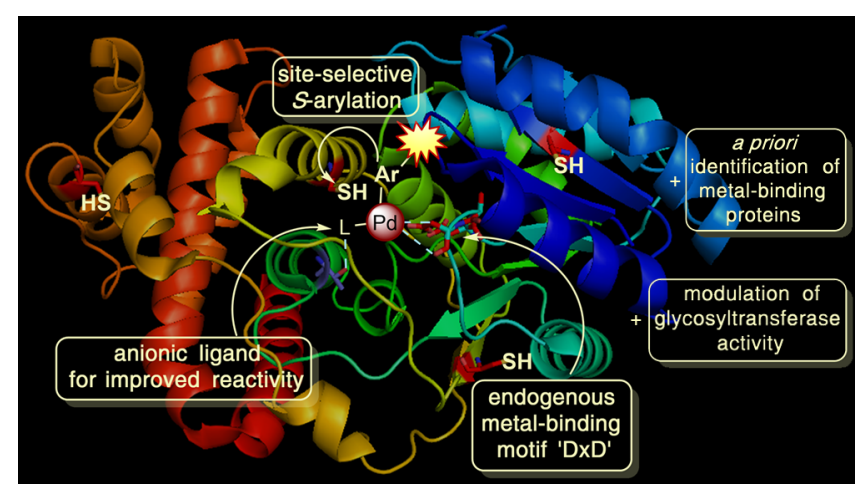

